\title{
Differing Effects of Education on Cognitive Decline in Diverse Elders With Low Versus High Educational Attainment
}

\author{
Laura B. Zahodne, Yaakov Stern, and Jennifer J. Manly \\ Columbia University
}

\begin{abstract}
Objective: In light of growing debate over whether and how early life educational experiences alter late-life cognitive trajectories, this study sought to more thoroughly investigate the relationship between educational attainment and rates of late-life cognitive decline in a racially, ethnically, and educationally diverse population. Method: Older adults $(N=3,435)$ in the community-based Washington HeightsInwood Columbia Aging Project were administered neuropsychological tests of memory, language, visuospatial function, and processing speed at approximate 24-month intervals for up to 18 years. Second-order latent growth curves estimated direct and indirect (through income) effects of educational attainment on rates of global cognitive decline separately in individuals with low ( $0-8$ years) and high (9-20 years) educational attainment. Results: More years of education were associated with higher cognitive level and slower cognitive decline in individuals with low or high educational attainment. The association between having more than 9 years of education and exhibiting slower cognitive decline was fully mediated by income. Although having additional years of education up to 8 years was also associated with higher income, this did not explain associations between education and cognitive change in the low-education group. Conclusions: Early education (i.e., up to 8 years) may promote aspects of development during a sensitive period of childhood that protect against late-life cognitive decline independent of income. In contrast, later education (i.e., 9 years and beyond) is associated with higher income, which may influence late-life cognitive health through multiple, nonmutually exclusive pathways.
\end{abstract}

Keywords: cognitive aging, socioeconomic status, income, statistical modeling

Educational attainment is a key component of successful cognitive aging and a major protective factor in dementia (Stern, 2009). Recently, there has been growing debate over whether and how early life educational experiences alter late-life cognitive trajectories. As summarized by Anstey and Christensen (2000), many studies published in the 1990s analyzed two occasions of measurement and documented an association between more years of education and attenuated rates of late-life cognitive decline

This article was published Online First September 15, 2014.

Laura B. Zahodne, Yaakov Stern, and Jennifer J. Manly, Cognitive Neuroscience Division, Department of Neurology and Taub Institute for Research on Alzheimer's Disease and the Aging Brain, Columbia University.

This longitudinal study is based on data from the Washington HeightsInwood Columbia Aging Project (Tang et al., 2001; Manly et al., 2005). This research was supported in part by Grants AG037212 and AG000261 from the National Institute on Aging. The content is solely the responsibility of the authors and does not necessarily represent the official views of the National Institutes of Health. The sponsors had no role in the study design, data collection, analysis or interpretation, writing of the report, or decision to submit the article for publication.

Correspondence concerning this article should be addressed to Laura B. Zahodne, Cognitive Neuroscience Division, Department of Neurology and Taub Institute for Research on Alzheimer's Disease and the Aging Brain, Columbia University College of Physicians and Surgeons, 630 West 168th Street, P \& S Box 16, New York, NY 10032. E-mail: lbz2105@columbia.edu
(Albert et al., 1995; Arbuckle, Maag, Pushkar, \& Chaikelson, 1998; Butler, Ashford, \& Snowdon, 1996; Evans et al., 1993; Farmer, Kittner, Rae, Bartko, \& Regier, 1995). As summarized by Zahodne et al. (2011), more recent studies using three or more occasions of measurement and growth curve modeling have failed to identify this association (Christensen et al., 2001; Karlamangla et al., 2009; Tucker-Drob, Johnson, \& Jones, 2009; Van Dijk, Van Gerven, Van Boxtel, Van der Elst, \& Jolles, 2008; Wilson et al., 2009; Piccinin et al., 2013).

In explaining these discrepant results, researchers have highlighted analytic differences across studies (Piccinin et al., 2013; Zahodne et al., 2011). For example, many early studies using regression-based approaches conditioned on the baseline measure of cognition. Conditioning on baseline cognitive level has been shown to produce biased effect estimates when the variable of interest is strongly related to baseline cognitive level, as is certainly the case with years of education (Glymour, Weuve, Berkman, Kawachi, \& Robins, 2005). Piccinin et al. (2013) recently addressed the issue of analytic variability in the literature by reporting results from six independent longitudinal studies of aging using a common analytic approach (i.e., growth curve model with time-in-study time basis) with a common set of predictors (i.e., age, sex, years of education) centered at common values. In that study, no association between education and cognitive decline was found for four of the six studies. In contrast, higher education was associated with slower rates of cognitive decline in the Origins of Variance in the Oldest-Old data set (Cederlöf \& Lorich, 1978; Pedersen, Lichtenstein, \& 
Svedberg, 2002), which featured relatively low average education (i.e., 7 years). Finally, education near the median (11 years), but not additional years, was associated with slower cognitive decline in the Canberra Longitudinal Study data set (Christensen et al., 2004). These results present the intriguing possibility that only early education influences late-life cognitive trajectories, whereas additional years of education beyond some minimum confer no additional protection. For example, Lyketsos, Chen, and Anthony (1999) proposed that additional education beyond 9 years is not associated with additional reduction in cognitive decline.

Another issue regarding the association between education and late-life cognitive decline that has been relatively overlooked by the epidemiologic literature relates to potential mechanisms. For example, education may influence later life cognitive trajectories by promoting aspects of cognitive and neural development during a sensitive period of childhood that protect against late-life cognitive decline. Another (nonmutually exclusive) possibility is that educational attainment sets an individual up for a lifetime of advantageous experiences, both cognitive (e.g., cognitively demanding occupation and leisure activities) and noncognitive (e.g., higher income, better quality neighborhoods, increased access to high-quality health care).

The overall goal of this study was to more thoroughly investigate the relationship between education and the rate of late-life cognitive decline in a racially, ethnically, and educationally diverse population. Specific aims were to (a) quantify the relationship between years of education and late-life cognitive decline; (b) determine whether years of education are only associated with late-life cognitive decline among individuals with relatively lower education; and (c) test whether associations between education and late-life cognitive decline are independent of or driven by lifetime advantage, as defined by late-life income.

\section{Method}

\section{Participants and Procedure}

The 3,435 older adults in this sample were participants in the Washington Heights-Inwood Columbia Aging Project (WHICAP). WHICAP is a prospective, community-based longitudinal study of aging and dementia in northern Manhattan, New York. Full descriptions of study procedures have been reported previously (Manly et al., 2005; Tang et al., 2001). In brief, individuals within the geographic area of northern Manhattan were identified from Medicare records and recruited in two waves: $1992(n=1,486)$ and 1999 ( $n=1,949)$. Among individuals who were contacted, $27 \%$ did not wish to participate in the baseline visit (Manly et al., 2005). The current sample included only participants who did not meet Diagnostic and Statistical Manual of Mental Disorders (3rd ed., rev.; DSM-III) criteria for dementia at their first neuropsychological evaluation, according to a consensus group of neurologists, psychiatrists, and neuropsychologists. Of the 3,444 participating individuals who did not meet criteria for dementia at baseline, information for years of education was unavailable for nine $(0.3 \%)$. The remaining 3,435 individuals were included in the present sample. Attempts at follow-up confirmed that $210(6 \%)$ died before the first follow-up visit. Of the 3,225 presumed eligible for follow-up, 2,604 (81\%) participated in at least one follow-up visit.

Ongoing follow-up at the 18- to 24-month interval included a battery of cognitive, functional, and health measures administered in the participant's preferred language (English or Spanish). This study complied with the ethical rules for human experimentation stated in the Declaration of Helsinki, including approval of the local institutional review board and informed consent. Baseline characteristics of the sample are shown in Table 1.

Table 1

Sample Characteristics at Baseline

\begin{tabular}{lccc}
\hline \multicolumn{1}{c}{ Variable } & $\begin{array}{c}\text { Low education } \\
(0-8 \text { years; } n=1,419)\end{array}$ & $\begin{array}{c}\text { High education } \\
(9-20 \text { years; } n=2,016)\end{array}$ & $p$ \\
\hline Mean $(S D)$ birth year & $1920(7.4)$ & $1922(7.2)$ & $<.001$ \\
Mean $(S D)$ age (years) & $76.4(6.4)$ & $76.2(6.5)$ & .40 \\
Sex $\%$ female) & 67.5 & 67.4 & .93 \\
Race $(\%$ Black) & 20.6 & 41.7 & $<.001$ \\
Ethnicity (\% Hispanic) & 71.2 & 17.4 & $<.001$ \\
Mean (SD) Education & $5.0(2.6)$ & $13.1(2.6)$ & $<.001$ \\
Recruitment year $(\%$ 1992) & 53.7 & 35.9 & $<.001$ \\
Mean $(S D)$ monthly income $(1-12)$ & $3.5(2.1)$ & $6.5(3.2)$ & $<.001$ \\
Mean $(S D)$ illness burden $(0-15)$ & $1.8(1.5)$ & $1.9(1.5)$ & .26 \\
Mean $(S D)$ Body mass index & $28.1(5.3)$ & $27.7(6.2)$ & .18 \\
Mean $(S D)$ CESD score $(0-10)$ & $2.1(2.2)$ & $1.7(1.9)$ & $<.001$ \\
Mean $(S D)$ baseline cognition ${ }^{\mathrm{a}}$ & & & \\
Memory & $47.2(7.6)$ & $52.1(8.4)$ & $<.001$ \\
Language & $46.6(8.0)$ & $52.6(5.9)$ & $<.001$ \\
Visuospatial & $45.5(9.1)$ & $53.3(6.3)$ & $<.001$ \\
Processing speed & $54.0(8.3)$ & $47.5(8.1)$ & $<.001$ \\
\hline
\end{tabular}

Note. $\quad$ CESD $=10$-item version of the Center for Epidemiological Studies Depression Scale.

${ }^{\mathrm{a}}$ Cognitive composites are average $\mathrm{T}$ scores (unadjusted for demographics). ${ }^{\mathrm{b}}$ Higher scores correspond to worse performance on the processing speed composite. 


\section{Outcomes}

Neuropsychological functioning was assessed with a comprehensive battery as previously described (Stern et al., 1992). Confirmatory factor analysis summarized this battery into four cognitive domains that are invariant across English and Spanish speakers: memory, language, visuospatial functioning, and processing speed (Siedlecki et al., 2010). Based on this factor analysis, composite scores were derived by converting all cognitive variables to $\mathrm{T}$ scores using means and standard deviations from this sample's initial visit and averaging resultant $\mathrm{T}$ scores within each of the four domains. In brief, memory composite scores included immediate, delayed, and recognition trials from the Selective Reminding Test (Buschke \& Fuld, 1974). Language scores included measures of naming, letter and category fluency, verbal abstract reasoning, repetition, and comprehension. Visuospatial scores included recognition and matching trials from the Benton Visual Retention Test (Benton, 1955), the Rosen Drawing Test (Rosen, 1981), and the Identities and Oddities subtest of the Dementia Rating Scale (Mattis, 1976). Processing speed scores included both trials of the Color Trails Test.

\section{Covariates}

Initial models controlled for birth cohort, baseline age, sex, race, ethnicity, education, and recruitment year. Birth cohort was determined by birth year, separated into 10-year bands: 1900-1909, 1910-1919, 1920-1929, 1930-1939, and 1940-1949. Sex, race, and ethnicity were determined via self-report using the format of the 2000 U.S. Census. Education (0-20 years) was measured via self-report. Subsequent models additionally included monthly household income at baseline. Income was measured in $86 \%$ of the sample via self-report using the following 12 categories: $\$ 450$ or less, \$451-\$550, \$551-650, \$651-750, \$751-\$1,000, \$1,001$\$ 1,250, \$ 1,251-\$ 1,500, \$ 1,501-\$ 1,750, \$ 1,751-\$ 2,000, \$ 2,001-$ $3000, \$ 3,001-\$ 4,000$, and more than $\$ 4,000$. To facilitate parameter interpretation, we centered covariates such that values of 0 corresponded to age 76, birth cohort of 1900-1909, male sex, White race, non-Hispanic ethnicity, 0 years of education, recruitment in 1992, and monthly income of $\$ 450$ or less, unless otherwise stated.

Additional models were stratified by dementia conversion status. Specifically, individuals who converted to DSM-III dementia based on consensus conference at any visit subsequent to the baseline visit were classified as "converters." Health status and depressive symptoms were also examined as potential components of the pathway leading from education to late-life cognitive decline. As described previously (Zahodne, Stern, \& Manly, 2014), overall illness burden was quantified by summing the self-reported number of the following conditions: heart disease, hypertension, stroke, diabetes, pulmonary disease, thyroid disease, liver disease, renal insufficiency, peptic ulcer disease, peripheral vascular disease, cancer, Parkinson's disease, essential tremor, multiple sclerosis, and arthritis. Depressive symptoms were quantified with a 10-item version of the Center for Epidemiological Studies Depression Scale (Irwin, Artin, \& Oxman, 1999). Maximum scores on the illness burden and depressive symptom measures observed during the study period were used.

\section{Statistical Analysis}

Descriptive statistics were computed using SPSS Version 19. Latent growth curve analysis was conducted in Mplus Version 7 using maximum likelihood estimation. Time was parameterized as years from study entry. The time score option in Mplus was used to accommodate participants' differing intervals between study visits. The average number of years between study visits throughout the entire study period was $2.18(S D=0.99)$. In total, five visits (follow-up up to 18 years) were analyzed to maximize covariance coverage. Missing data were managed with full information maximum likelihood using all available data at each occasion.

All longitudinal models were second-order latent growth curve models (i.e., curve of factors), as depicted in Figure 1. Latent factors representing general cognitive ability at each occasion reflect shared variance among the four cognitive composite scores described above. Factor loadings were forced to equivalence across visits (i.e., stationarity). Within each cognitive domain, measurement intercepts (i.e., intercepts in the regression models relating each cognitive composite score to the latent factor) were forced to equivalence across time. Residuals were freely estimated and allowed to correlate over time within cognitive domains. Functional form was determined in the entire sample by statistically comparing models allowing only linear change to models allowing both linear and quadratic change using the chi-square test.

Aim 1: Quantify the relationship between education and cognitive decline. Education and covariates (i.e., birth cohort, baseline age, sex, race, ethnicity, and recruitment year) were added to the best-fitting model in the entire sample. All growth parameters (i.e., initial cognitive level, annual rate of change, change in the rate of change) were regressed onto education and all covariates.

Aim 2: Determine whether education is only associated with cognitive decline in the low-education group. Stratified models estimated the effects of education on the growth parameters separately in individuals with low ( $0-8$ years) versus high (9-20 years) educational attainment. A cutoff point of 8 years was chosen for three reasons. First, Grade 9 marks meaningful transitions from childhood to adolescence in human development and from primary to secondary school in the United States. This cutoff point allowed for testing the hypothesis of qualitative differences between early (i.e., primary) and later (i.e., secondary and postsecondary) schooling. Second, the limit of 8 years falls within the range given by studies suggesting that only early education influences late-life cognitive trajectories (Lyketsos et al., 1999; Piccinin et al., 2013). Finally, having 10 years of education was the mean, median, and mode in this sample, but having 8 years of education reflects a second mode that also allowed for comparable sample sizes in low- and high-education groups. Importantly, results were unchanged when a cutoff point of 10 years was used.

All growth parameters were regressed onto education and all covariates. To facilitate parameter interpretation, we centered education at 9 years in the high-education group. Therefore, values of 0 correspond to 0 years of education in the low-education group and 9 years of education in the high-education group.

Aim 3: Test whether education-cognitive decline associations are driven by income. Income was added to Aim 2 models and regressed onto education. Initial models allowed both direct 


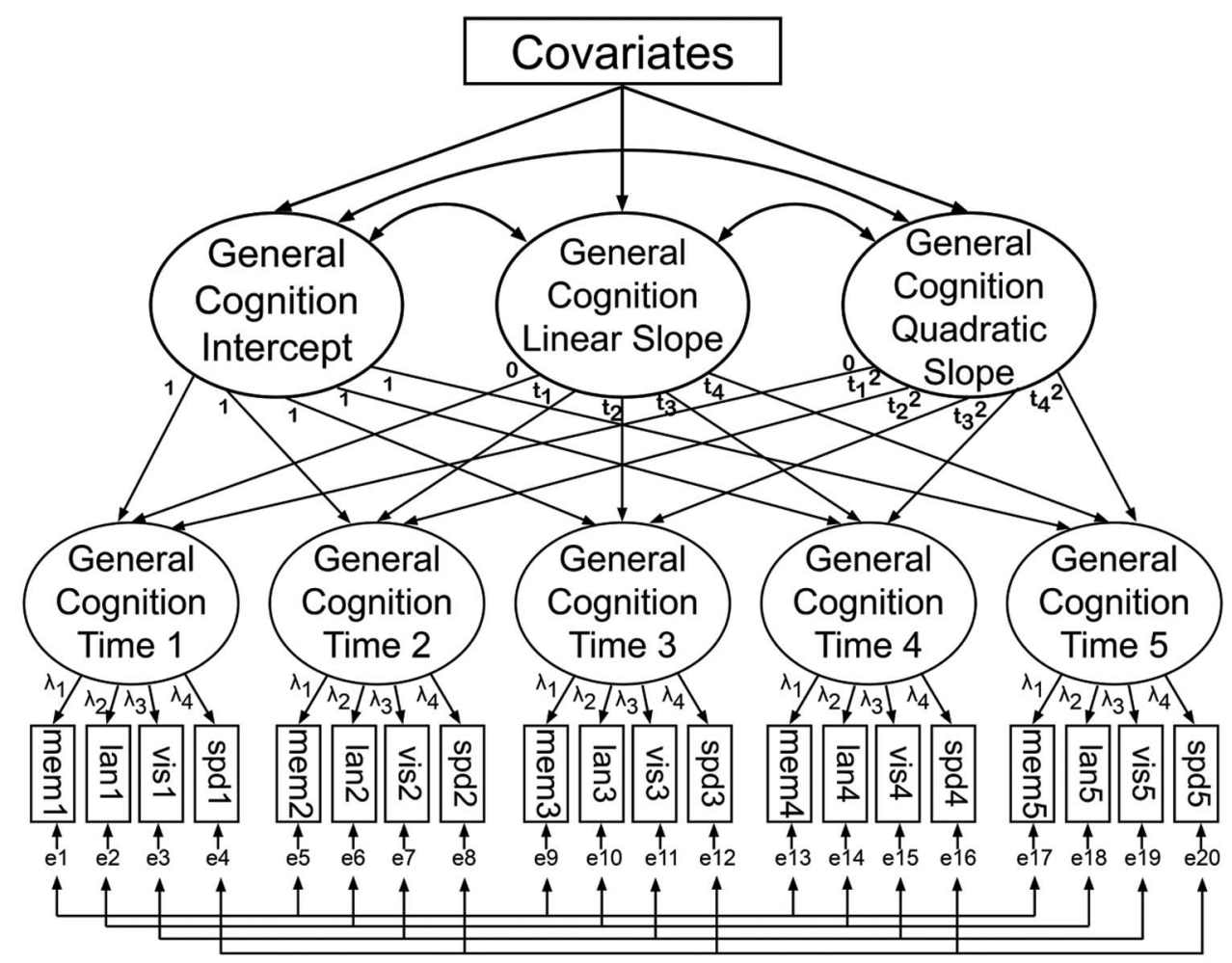

Figure 1. Schematic of the second-order latent growth curve model. Boxes represent observed variables and ellipses represent latent factors. First-order latent factors correspond to general cognitive ability at each occasion and reflect shared variance among the four cognitive composite scores measured at the respective occasion. First-order factor loadings $(\lambda)$ were forced to equivalence across time (i.e., stationarity). Within each cognitive domain, measurement intercepts (i.e., intercepts in the regression models relating each cognitive composite score to the latent factor) were also forced to equivalence across time. Residuals (e) were freely estimated and allowed to correlate over time within cognitive domains. Second-order factors correspond to the growth parameters. The latent intercept reflects initial cognitive level, the latent linear slope reflects rate of change, and the latent quadratic slope reflects changes in the rate of change (i.e., acceleration or deceleration). Time was parameterized as years from study entry, which was calculated separately for each participant to accommodate participants' differing intervals between study visits. Mem = memory composite; lan = language composite; vis = visuospatial composite; $\mathrm{spd}=$ speed composite.

and indirect (through income) effects of education on cognitive trajectories. All growth parameters were regressed onto education, income, and covariates. Chi-square tests compared fit between models allowing both direct and indirect effects of education on linear and quadratic change and models allowing only direct effects or indirect effects. When there was no significant difference in fit, the more parsimonious model (i.e., fewer parameters) was retained.

\section{Results}

\section{Differences Between Groups}

As shown in Table 1, individuals with low education (0-8 years) were born earlier, were more likely to be Hispanic, were more likely to be recruited in the earlier recruitment wave (1992), reported lower monthly income and a greater number of depressive symptoms, and scored worse on tests of memory, language, visuospatial functioning, and processing speed, compared with indi- viduals with high education (9-20 years). There were no differences between education groups in sex, baseline age, number of self-reported health conditions, or body mass index.

\section{Characterizing the Trajectories}

Compared with a model allowing only linear change, fit was improved by allowing curvilinear change $\left(\Delta \chi^{2}(4)=-27.13, p<\right.$ $.001)$. Therefore, models estimating both linear and quadratic slopes were retained for subsequent model building.

Aim 1: Quantify the relationship between education and cognitive decline. Next, the predictor of interest (education) and the relevant covariates described above were added to the model. Table 2 presents unstandardized estimates of the effects of these variables on the cognitive trajectories. As shown, higher education was associated with higher initial cognitive level and shallower cognitive decline over time, independent of all covariates. Figure 2 depicts model-estimated trajectories for three different education levels, controlling for covariates. As shown, education-related 
Table 2

Unstandardized Covariate Effects (Standard Errors) on Global Cognitive Trajectories in the Whole Sample

\begin{tabular}{lccc}
\hline \multicolumn{1}{c}{ Variable } & Initial level & Linear change & Quadratic change \\
\hline Birth cohort & $0.76(0.29)^{*}$ & $-0.14(0.11)$ & $0.02(0.01)$ \\
Age & $-0.18(0.03)^{* * *}$ & $-0.08(0.01)^{* * *}$ & $0.01(0.00)^{*}$ \\
Female sex & $0.50(0.21)^{*}$ & $-0.08(0.08)$ & $0.01(0.01)$ \\
Black & $-1.86(0.27)^{* * *}$ & $-0.22(0.11)^{*}$ & $0.02(0.01)$ \\
Hispanic & $-2.80(0.30)^{* * *}$ & $0.05(0.12)$ & $-0.00(0.01)$ \\
Recruitment in 1999 & $-0.39(0.30)$ & $0.00(0.11)$ & $-0.00(0.01)$ \\
Education & $0.70(0.03)^{* * *}$ & $0.03(0.01)^{*}$ & $-0.00(0.00)$ \\
\hline
\end{tabular}

${ }^{*} p<.05 .{ }^{* *} p<.001$.

differences in cognitive level are much more evident than education-related differences in rate of cognitive decline.

Aim 2: Determine whether education is only associated with cognitive decline in the low-education group. To examine the association between educational attainment and cognitive decline separately among individuals with high versus low education, we stratified the final model described above by educational level ( $0-8$ years vs. $9-20$ years). Independent of the covariates, higher education was associated with higher cognitive level in both loweducation $(B=0.745, S E=0.067, p<.001)$ and high-education groups $(B=0.690, S E=0.064, p<.001)$. In the low-education group, more years of education were associated with slower linear decline $(B=0.065, S E=0.027, p=.018)$ but accelerated quadratic decline $(B=-0.007, S E=0.003, p=.020)$. Figure 3 depicts model-estimated trajectories for different educational levels, controlling for covariates. As shown, an individual with 8 years of education is predicted to experience a longer period of maintenance of global cognitive functioning before evidencing decline, as compared with an individual with 0 years of education. In the high-education group, more years of education were associated with slower linear decline $(B=0.048, S E=0.022, p=$ $.032)$ but were not associated with quadratic decline $(B=-0.001$, $S E=0.002, p=.687$ ). As shown in Figure 3, an individual with 20 years of education is predicted to experience slower cognitive decline throughout the observation period, as compared with an individual with 9 years of education.

More individuals in the low-education group were diagnosed with dementia during the study period than individuals in the high-education group $\left(25.9 \%\right.$ vs. $12.0 \% ; \chi^{2}(1)=111.734 ; p<$ $.001)$. Among the relatively small number of "converters" ( $n=$ 602), the association between higher education and accelerated quadratic decline did not reach significance, but the magnitude of this association was similar to that in the low-education group $(B=-0.006, S E=0.004, p=.158)$. In contrast, there was no evidence of this association among the 2,767 "nonconverters" $(B=0.000, S E=0.001, p=.922)$.

Aim 3: Test whether education-cognitive decline associations are driven by income. To determine the extent to which the above-described associations were driven by lifetime advantage, we added income to the education group-stratified models and regressed it onto educational attainment. In models allowing both direct and indirect effects, all growth parameters were regressed onto education, income, and all covariates. In the loweducation group, there were direct effects of education on linear $(B=0.063, S E=0.028, p=.023)$ and quadratic $(B=-0.007$, $S E=0.003, p=.025)$ cognitive decline, independent of income. There were no indirect effects of education on cognitive decline. Specifically, higher education was associated with higher late-life income $(B=0.167, S E=0.023, p<.001)$, but late-life income was not associated with cognitive decline independent of the direct effects of education (linear: $B=0.021, S E=0.038, p=.581$; quadratic: $B=-0.002, S E=0.004, p=.651)$. A model allowing only indirect effects of education on linear and quadratic cognitive decline fit significantly worse than the model allowing both direct and indirect effects $\left(\chi^{2}(2)=6.546, p<.05\right)$. In contrast, a model allowing only direct effects of education on linear and quadratic cognitive decline did not fit significantly worse than the model allowing both direct and indirect effects $\left(\chi^{2}(2)=0.322, p>.05\right)$. Therefore, the more parsimonious model allowing only direct effects of education on cognitive decline was retained and is summarized in Figure 4.

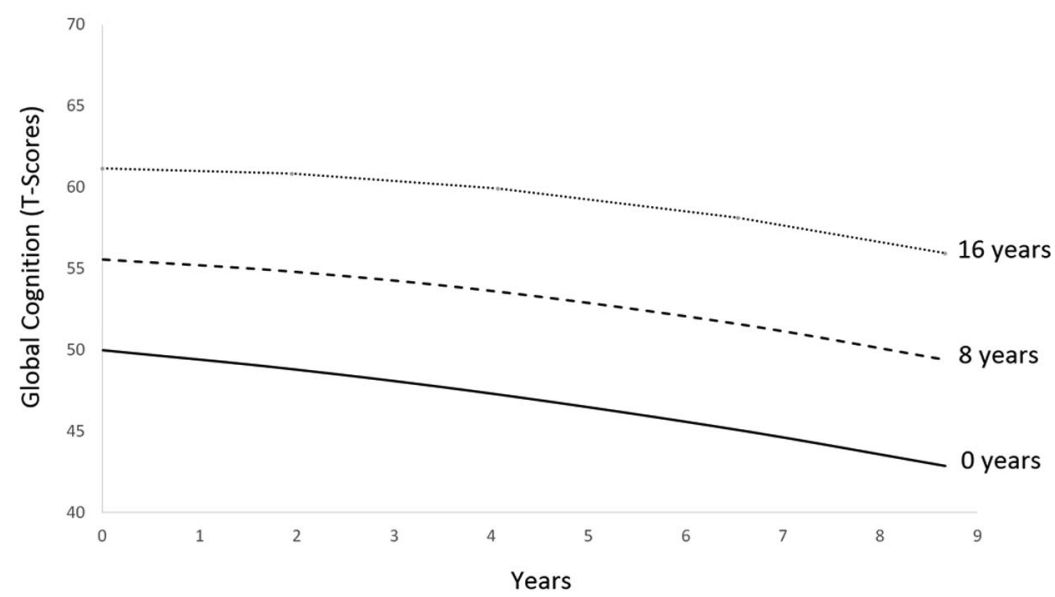

Figure 2. Model-estimated cognitive trajectories for 76-year-old, White, non-Hispanic men born 1900-1909, recruited in 1992 , with 0,8 , or 16 years of education. 


\section{A. Low Education}

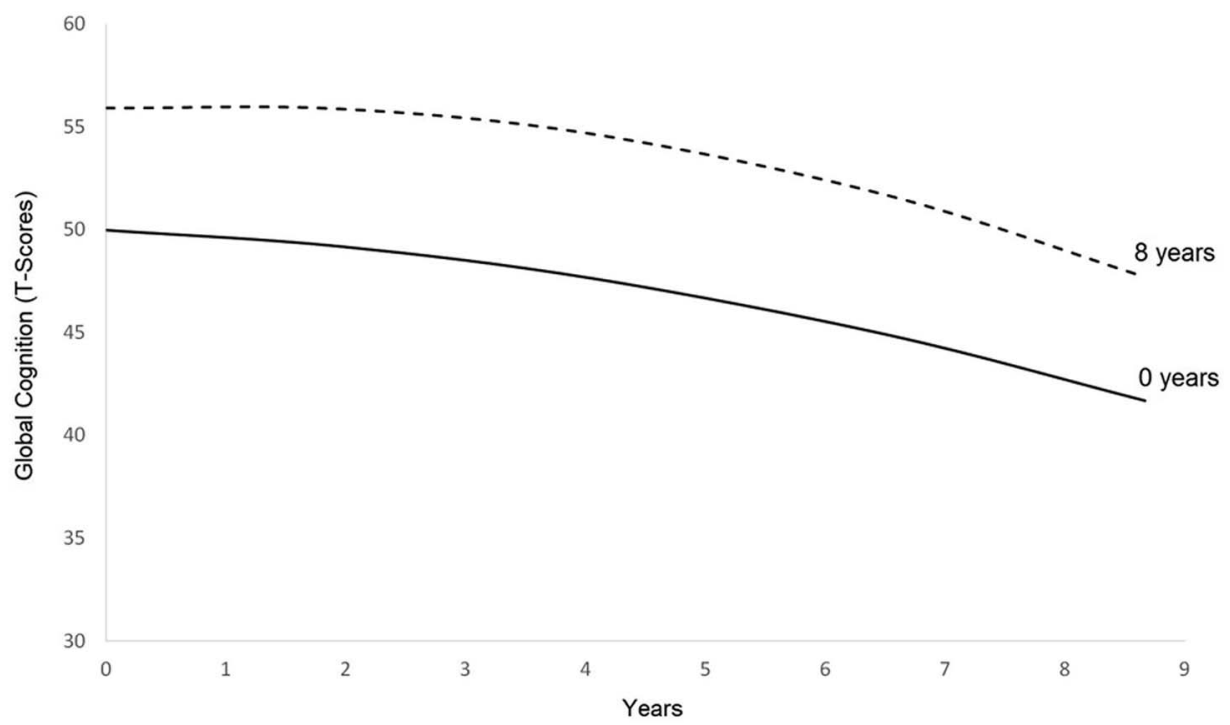

B. High Education

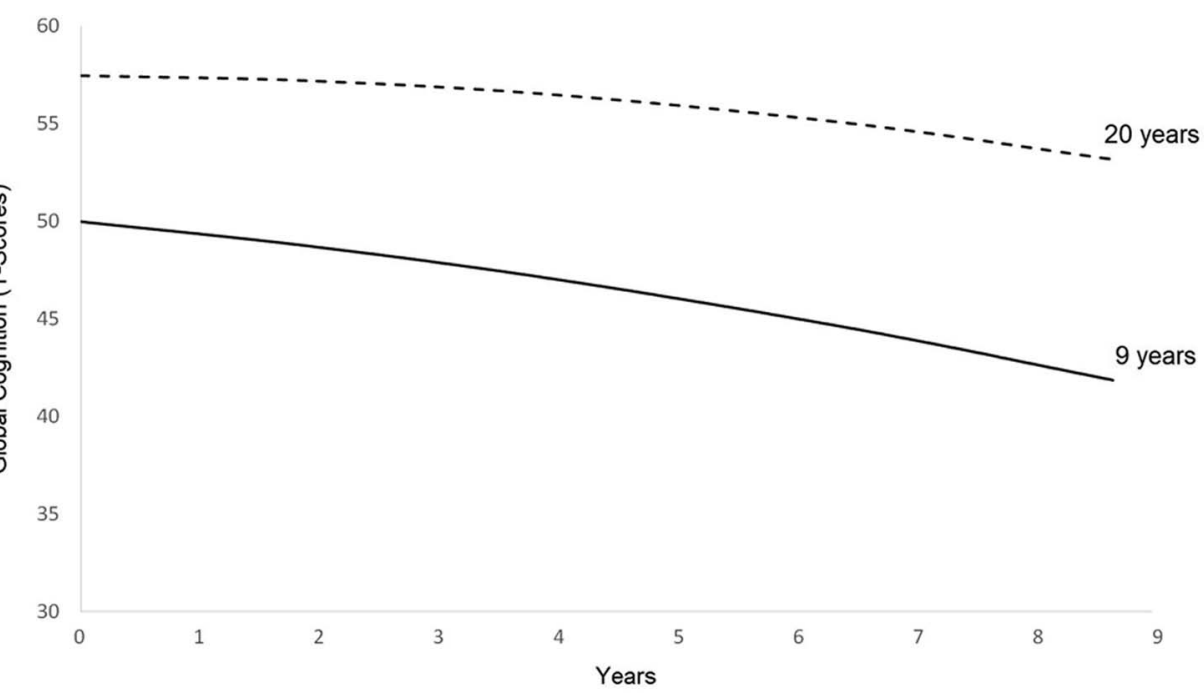

Figure 3. Model-estimated cognitive trajectories for 76-year-old, White, non-Hispanic men born 1900-1909, recruited in 1992, with low (0-8 years) or high (9-20 years) education. Lines correspond to the extremes of educational attainment in the low- and high-education groups. In the low-education group (A), higher education was associated with attenuated linear decline but accelerated quadratic decline. In other words, trajectories first diverge and then converge over the course of the study period. In the high-education group (B), higher education was associated with attenuated linear decline, as shown by divergence of the lines over the entire study period.

In a model estimating both direct and indirect effects within the high-education group, neither education (linear: $B=0.037, S E=$ $0.024, p=.122$; quadratic: $B=-0.002, S E=0.003, p=.536$ ) nor income (linear: $B=0.031, S E=0.023, p=.168$; quadratic: $B=0.001, S E=0.002, p=.656$ ) was independently associated with cognitive decline. A subsequent model allowing only direct effects of education on cognitive decline fit significantly worse than the model allowing both direct and indirect effects $\left(\chi^{2}(2)=\right.$ 15.938, $p<.001)$. A subsequent model allowing only indirect effects of education on cognitive decline (through income) did not fit significantly worse than the model allowing both direct and indirect effects $\left(\chi^{2}(2)=4.978, p>.05\right)$. Therefore, the model allowing only indirect effects of education on cognitive decline was retained and is shown in Figure 4. As shown, higher education was associated with higher income, which in turn was associated with less linear cognitive decline. There was no association between income and quadratic cognitive decline $(B=0.001, S E=$ $0.002, p=.816)$.

To explore the role of health factors, we added illness burden and depressive symptoms as predictors of the growth parameters in the best-fitting models and regressed these variables on income. The direct effects of education on cognitive decline 
A. Low Education

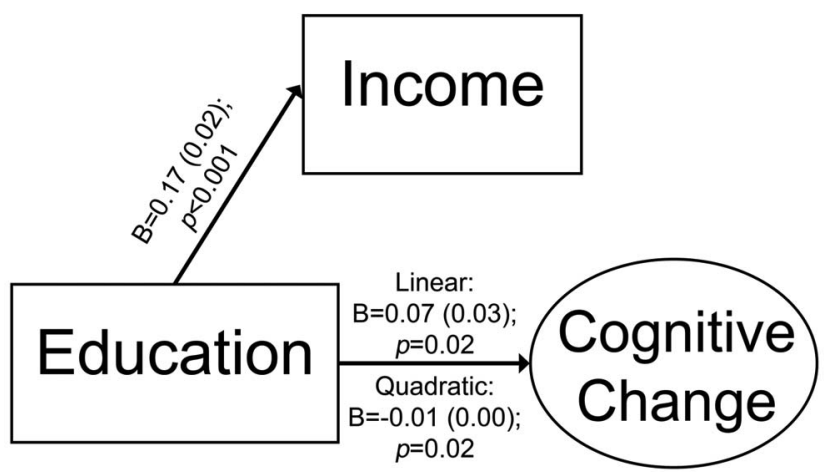

B. High Education

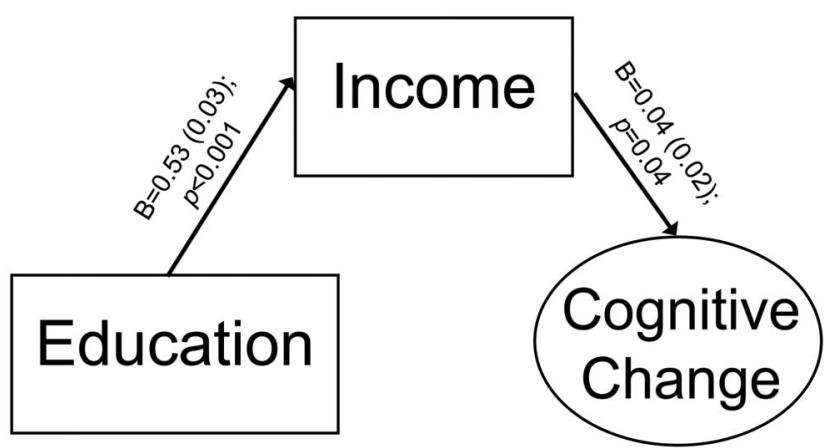

Figure 4. Schematic of final models examining direct and indirect (through income) effects of education on cognitive change for individuals with low ( $0-8$ years) or high (9-20 years) education. For simplicity, covariates and the other growth parameters are not shown.

remained significant in the low-education group, and there were no independent effects of either illness burden or depressive symptoms on decline. Similarly, the indirect effect of education (through income) on linear cognitive decline remained significant in the high-education group, and there were no independent effects of either illness burden or depressive symptoms on decline.

\section{Discussion}

In this sample of racially, ethnically, and educationally diverse older adults living in northern Manhattan, more years of education were associated with higher cognitive level and slower cognitive decline. These effects were observed both for individuals with relatively low $(0-8)$ and relatively high (9-20) educational attainment. However, follow-up analyses suggest different interpretations of associations involving additional education up to 8 years versus additional education beyond 9 years. Specifically, the association involving more than 9 years of education was fully mediated by income, a proxy for lifetime advantage. Although having additional years of education up to 8 years was also associated with higher income, this did not explain the association between education and cognitive change in the low-education group.
The finding that more early education (i.e., additional years up to 8) is associated with slower late-life cognitive decline independent of later income, illness burden, and depressive symptoms suggests that protection may be conferred through an alternative mechanism to lifetime advantage. Specifically, early education may promote aspects of cognitive and neural development during a sensitive period of childhood (Hertzman, 1994). It is also possible that the content of early education is more relevant than the timing. Early education may instill certain basic skills that protect against late-life cognitive declines. The newly emerging field of educational neuroscience is beginning to demonstrate how educational experiences shape the brain in enduring ways (Hinton, Miyamoto, \& Della-Chiesa, 2008).

In contrast, later education (i.e., additional years beyond 9) was not associated with later cognitive decline independent of its association with income. In this sample, later education was associated with higher income, which may have influenced late-life cognitive health through multiple, nonmutually exclusive pathways (e.g., increasing access to high-quality health care, decreasing exposure to stressors, increasing participation in cognitively complex occupations and/or leisure activities). However, additional analyses indicated that neither health (i.e., self-reported illnesses and depressive symptoms) variables nor the total number of leisure activities done in the past month (Helzner, Scarmeas, Cosentino, Portet, \& Stern, 2007) explained the association between income and cognitive decline. The relationship between educational attainment and income was weaker in the loweducation group compared with the high-education group. This finding was also evidenced by a wider distribution of income in the high-education group compared with the low-education group. Thus, additional years of education up to 8 may not have as broad an influence on aspects of lifetime advantage as additional years of education beyond 9 .

Higher cognitive level among individuals with higher educational attainment is a near-ubiquitous finding in the aging literature. However, the present finding that higher educational attainment is also associated with slowed linear cognitive decline contrasts several recent longitudinal studies that have used growth curve models to estimate cognitive change (Christensen et al., 2001; Karlamangla et al., 2009; Tucker-Drob et al., 2009; Van Dijk et al., 2008; Wilson et al., 2009; Zahodne et al., 2011). One important difference between this study and previous studies is the racial, ethnic, and educational diversity of the WHICAP cohort. Interestingly, another study with strong representation of individuals with relatively low educational attainment also reported an association between education and cognitive decline (Piccinin et al., 2013). However, this result was also true when analyses were restricted to WHICAP participants with relatively high education (9-20 years), which suggests that the observed association between educational attainment and cognitive decline was not driven by educational disadvantage.

Another difference from previous studies is the use of secondorder latent growth curve models to estimate global cognitive changes. Second-order latent growth curve models separate observed variance in a cognitive score into reliable construct variance, reliable unique variance, and random occasion-specific variance (Sayer \& Cumsille, 2001). Removing measurement error from the latent growth parameters disattenuates regression coefficients representing associations between the growth parameters 
and variables of interest and produces smaller accompanying standard errors. Therefore, second-order latent growth curve models theoretically provide more precise estimates of the association between education and global cognitive change. In contrast, most previous studies employed first-order latent growth curve models or mixed-effects models, which confound multiple sources of variance in a cognitive score. It should be noted that one previous study that used second-order latent growth curve models to examine the association between education and cognitive change included latent factors only indicated by measures of crystallized intelligence (e.g., vocabulary, reading) and relatively simple memory tasks designed to detect dementia (e.g., three-item recall from the Mini-Mental State Exam, address recall from the Clinical Dementia Rating), both of which exhibit relatively little change with normal aging (Christensen et al., 2001). The current study included a comprehensive neuropsychological battery with multiple measures that are more sensitive to age-related changes. It should be emphasized that the magnitude of the association between education and rate of cognitive change was quite small, particularly in relation to the magnitude of the association between education and cognitive level. It is possible that sensitive measures and use of second-order latent growth curve modeling allowed us to detect this small association.

Among individuals with relatively low education ( $0-8$ years), additional years of education were associated with slower linear decline coupled with accelerated quadratic decline. In other words, more early education was associated with a longer period of relative maintenance of cognitive functioning. Follow-up analyses revealed that this association between higher education and accelerated quadratic decline was only evident in individuals who converted to dementia. Thus, this finding is in line with the somewhat paradoxical phenomena of accelerated decline among individuals with dementia and high cognitive reserve compared with low cognitive reserve (Stern, 2009). Indeed, several reports indicate that individuals with higher education exhibit steeper cognitive trajectories following the diagnosis of dementia (Andel, Vigen, Mack, Clark, \& Gatz, 2006; Hall et al., 2007; Stern, Albert, Tang, \& Tsai, 1999). It is likely that this finding was detected only in the low-education group because significantly more individuals in that group were diagnosed with dementia during this study period, in line with substantial research showing that lower educational attainment is a consistently strong predictor of earlier dementia diagnosis (Stern, 2012).

Limitations of this study include relatively low representation of Hispanics in the high-education group. Because ethnicity was not associated with cognitive decline in a model that excluded education, it is unlikely that the different pattern of results in the two education groups was driven by differences in the distribution of ethnicity. Furthermore, the difference in proportions of Hispanics versus non-Hispanic Whites reporting $\leq 8$ years of education in the current sample ( $71.2 \%$ vs. $12.5 \%$ ) is comparable to the difference in proportions in the general U.S. population of adults aged 65 and older (36.2\% vs. $5.0 \%$; U.S. Census, 2013). It should be noted that the current sample included a higher proportion of individuals reporting $\leq 8$ years of education $(41.3 \%)$ than in the general U.S. population of adults aged 65 and older (8.4\%; U.S. Census, 2013).

Another limitation is the lack of a high-quality measure of occupational complexity to estimate lifetime advantage more comprehensively. Additional analyses (data not shown) did not reveal a relationship between a basic measure of occupational status (i.e., unskilled/semiskilled, skilled, clerical/office, managerial, professional/technical) and cognitive change. However, an occupational measure reflecting the specific cognitive demands of different occupations may have yielded different results. Future studies should quantify the cognitive complexity of participants' occupations and explore the interplay between education, income, and different aspects of occupation in predicting late-life cognitive trajectories. Finally, a limitation of this and all observational studies of cognitive aging is the inability to rule out the possibility that innate abilities represent a common cause of both educational attainment and attenuated cognitive decline (Deary \& Johnson, 2010).

Strengths of this study over previous work include the racial, ethnic, and educational diversity of this sample, the communitybased sampling approach of WHICAP, use of second-order growth curve models to more precisely estimate late-life cognitive change, and tests of mediation of effects by income.

In conclusion, early education (i.e., additional years up to 8) is associated with late-life cognitive change, independent of income. In contrast, the association between later education (i.e., additional years beyond 9) and late-life cognitive change was not independent of income. These results suggest that later education leads to higher income, which may influence late-life cognitive health through multiple, nonmutually exclusive pathways (e.g., increasing access to high-quality health care, decreasing exposure to stressors, increasing participation in more cognitively demanding occupations and/or leisure activities). Measures of self-reported illness burden and depressive symptoms could not explain the effects of income on late-life cognitive decline, and future research should explore these pathways with a wider range of sensitive measures.

\section{References}

Albert, M. S., Jones, K., Savage, C. R., Berkman, L., Seeman, T., Blazer, D., \& Rowe, J. W. (1995). Predictors of cognitive change in older persons: MacArthur studies of successful aging. Psychology and Aging, 10, 578-589. doi:10.1037/0882-7974.10.4.578

Andel, R., Vigen, C., Mack, W. J., Clark, L. J., \& Gatz, M. (2006). The effect of education and occupational complexity on rate of cognitive decline in Alzheimer's patients. Journal of the International Neuropsychological Society, 12, 147-152. doi:10.1017/S1355617706060206

Anstey, K., \& Christensen, H. (2000). Education, activity, health, blood pressure and apolipoprotein $\mathrm{E}$ as predictors of cognitive change in old age: A review. Gerontology, 46, 163-177. doi:10.1159/000022153

Arbuckle, T. Y., Maag, U., Pushkar, D., \& Chaikelson, J. S. (1998). Individual differences in trajectory of intellectual development over 45 years of adulthood. Psychology and Aging, 13, 663-675. doi:10.1037/ 0882-7974.13.4.663

Benton, A. L. (1955). The Visual Retention Test. New York, NY: Psychological Corporation.

Buschke, H., \& Fuld, P. A. (1974). Evaluating storage, retention, and retrieval in disordered memory and learning. Neurology, 24, 1019-1025. doi:10.1212/WNL.24.11.1019

Butler, S. M., Ashford, J. W., \& Snowdon, D. A. (1996). Age, education, and changes in the Mini-Mental State Exam scores of older women: Findings from the Nun Study. Journal of the American Geriatric Society, 44, 675-681.

Cederlöf, R., Lorich, U. (1978). The Swedish Twin Registry. In W. E. Nance, G. Allen, \& P. Parisi (Eds.), Twin research: Part C, Biology and epidemiology (pp. 189-195). New York, NY: Alan R. Liss. 
Christensen, H., Hofer, S. M., MacKinnon, A. J., Korten, A. E., Jorm, A. F., \& Henderson, A. S. (2001). Age is no kinder to the better educated: Absence of an association investigated using latent growth techniques in a community sample. Psychological Medicine, 31, 15-28. doi:10.1017/S0033291799002834

Christensen, H., Mackinnon, A., Jorm, A. F., Korten, A., Jacomb, P., Hoger, S. M., \& Henderson, S. (2004). The Canberra Longitudinal Study: Design, aims, methodology, outcomes and recent empirical investigations. Aging, Neuropsychology, and Cognition, 11, 169-195. doi: $10.1080 / 13825580490511053$

Deary, I. J., \& Johnson, W. (2010). Intelligence and education: Causal perceptions drive analytic processes and therefore conclusions. International Journal of Epidemiology, 39, 1362-1369. doi:10.1093/ije/dyq072

Evans, D. A., Becket, L. A., Albert, M. S., Hebert, L. E., Scher, P. A., Funkenstein, H. H., \& Taylor, J. O. (1993). Level of education and change in cognitive function in a community population of older persons. Annals of Epidemiology, 3, 71-77. doi:10.1016/10472797(93)90012-S

Farmer, M. E., Kittner, S. J., Rae, D. S., Bartko, J. J., \& Regier, D. A. (1995). Education and change in cognitive function. The Epidemiologic Catchment Area Study. Annals of Epidemiology, 5, 1-7. doi:10.1016/ 1047-2797(94)00047-W

Glymour, M. M., Weuve, J., Berkman, L. F., Kawachi, I., \& Robins, J. M. (2005). When is baseline adjustment useful in analyses of change? An example with education and cognitive change. American Journal of Epidemiology, 162, 267-278. doi:10.1093/aje/kwi187

Hall, C. B., Derby, C., LeValley, A., Katz, M. J., Verghese, J., \& Lipton, R. B. (2007). Education delays accelerated decline on a memory test in persons who develop dementia. Neurology, 69, 1657-1664. doi:10.1212/ 01.wnl.0000278163.82636.30

Helzner, E. P., Scarmeas, N., Cosentino, S., Portet, F., \& Stern, Y. (2007). Leisure activity and cognitive decline in incident Alzheimer disease. Archives of Neurology, 64, 1749-1754.

Hertzman, C. (1994). The lifelong impact of childhood experiences: A population health perspective. Daedalus, 123, 167-180.

Hinton, C., Miyamoto, K., \& Della-Chiesa, B. (2008). Brain research, learning and emotions: Implications for education research, policy and practice. European Journal of Education, 43, 87-103. doi:10.1111/j .1465-3435.2007.00336.x

Irwin, M., Artin, K. H., \& Oxman, M. N. (1999). Screening for depression in the older adult: Criterion validity of the 10-item Center for Epidemiological Studies Depression Scale (CES-D). Archives of Internal Medicine, 159, 1701-1704.

Karlamangla, A. S., Miller-Martinez, D., Aneshensel, C. S., Seeman, T. E., Wight, R. G., \& Chodosh, J. (2009). Trajectories of cognitive function in late life in the United States: Demographic and socioeconomic predictors. American Journal of Epidemiology, 170, 331-342. doi:10.1093/ aje/kwp154

Lyketsos, C. G., Chen, L.-S., \& Anthony, J. C. (1999). Cognitive decline in adulthood: An 11.5-year follow-up of the Baltimore Epidemiologic Catchment Area Study. The American Journal of Psychiatry, 156, 5865.

Manly, J. J., Bell-McGinty, S., Tang, M.-X., Schupf, N., Stern, Y., \& Mayeux, R. (2005). Implementing diagnostic criteria and estimating frequency of mild cognitive impairment in an urban community. Archives of Neurology, 62, 1739-1746. doi:10.1001/archneur.62.11.1739

Mattis, S. (Ed.). (1976). Mental status examination for organic mental syndrome in the elderly patient. New York, NY: Grune \& Stratton.

Pedersen, N. L., Lichtenstein, P., \& Svedberg, P. (2002). The Swedish Twin Registry in the third millennium. Twin Research, 5, 427-432. doi:10.1375/136905202320906219
Piccinin, A. M., Muniz, G., Clouston, S., Reynolds, C. A., Thorvaldsson, V., Deary, I. J., . . Hofer, S. M., (2013). Coordinated analysis of age, sex, and education effects on change in MMSE scores. The Journals of Gerontology, Series B: Psychological Sciences and Social Sciences, 68, 374-390, doi:10.1093/geronb/gbs077

Rosen, W. (1981). The Rosen Drawing Test. Bronx, NY: Veterans Administration Medical Center.

Sayer, A. G., \& Cumsille, P. E. (2001). Second-order latent growth models. In L. M. Collins \& A. G. Sayer (Eds.), New methods for the analysis of change (pp. 179-200). Washington, DC: American Psychological Association. doi:10.1037/10409-006

Siedlecki, K. L., Manly, J. J., Brickman, A. M., Schupf, N., Tang, M.-X., \& Stern, Y. (2010). Do neuropsychological tests have the same meaning in Spanish speakers as they do in English speakers? Neuropsychology, 24, 402-411. doi:10.1037/a0017515

Stern, Y. (2009). Cognitive reserve. Neuropsychologia, 47, 2015-2028. doi:10.1016/j.neuropsychologia.2009.03.004

Stern, Y. (2012). Cognitive reserve in ageing and Alzheimer's disease. Lancet Neurology, 11, 1006-1012. doi:10.1016/S1474-4422(12)70191-6

Stern, Y., Albert, S., Tang, M.-X., \& Tsai, W. Y. (1999). Rate of memory decline in $\mathrm{AD}$ is related to education and occupation: Cognitive reserve? Neurology, 53, 1942-1947. doi:10.1212/WNL.53.9.1942

Stern, Y., Andrews, H., Pittman, J., Sano, M., Tatemichi, T., Lantigua, R., \& Mayeux, R. (1992). Diagnosis of dementia in a heterogeneous population. Development of a neuropsychological paradigm-based diagnosis of dementia and quantified correction for the effects of education. Archives of Neurology, 49, 453-460. doi:10.1001/archneur.1992 .00530290035009

Tang, M.-X., Cross, P., Andrews, H., Jacobs, D. M., Small, S., Bell, K., . . Mayeux, R. (2001). Incidence of AD in African-Americans, Caribbean Hispanics, and Caucasians in northern Manhattan. Neurology, 56, 4956. doi:10.1212/WNL.56.1.49

Tucker-Drob, E. M., Johnson, K. E., \& Jones, R. N. (2009). The cognitive reserve hypothesis: A longitudinal examination of age-associated declines in reasoning and processing speed. Developmental Psychology, 45, 431-446. doi:10.1037/a0014012

U.S. Census. (2013). Educational attainment. Retrieved from https://www .census.gov/hhes/socdemo/education/

Van Dijk, K. R., Van Gerven, P. W., Van Boxtel, M. P., Van der Elst, W., \& Jolles, J. (2008). No protective effects of education during normal cognitive aging: Results from the 6-year follow-up of the Maastricht Aging Study. Psychology and Aging, 23, 119-130. doi:10.1037/08827974.23.1.119

Wilson, R. S., Hebert, L. E., Scher, P. A., Barnes, L. L., Mendes de Leon, C. F., \& Evans, D. A. (2009). Educational attainment and cognitive decline in old age. Neurology, 72, 460-465. doi:10.1212/01.wnl $.0000341782 .71418 .6 \mathrm{c}$

Zahodne, L. B., Glymour, M. M., Sparks, C., Bontempo, D., Dixon, R. A., MacDonald, S. W. S., \& Manly, J. J. (2011). Education does not slow cognitive decline with aging: 12-year evidence from the Victoria Longitudinal Study. Journal of the International Neuropsychological Society, 17, 1039-1046. doi:10.1017/S1355617711001044

Zahodne, L. B., Stern, Y., \& Manly, J. J. (2014). Depressive symptoms precede memory decline, but not vice versa, in non-demented older adults. Journal of the American Geriatrics Society, 62, 130-134. doi 10.1111 /jgs. 12600

Received May 1, 2014

Revision received August 5, 2014 Accepted August 5, 2014 